\title{
Development and Evaluation of a Water-in-oil Microemulsion Formulation for the Transdermal Drug Delivery of Teriflunomide (A771726)
}

\author{
Yaru Cao, ${ }^{a, b}$ Huifang Gao, ${ }^{a, b}$ Hongguang Xia,${ }^{a, b}$ Xiangyu Zhu, ${ }^{a, b}$ Biao Li,${ }^{a, b}$ Xuya Zhou, ${ }^{c}$ and \\ Yong $\operatorname{Jin}^{*, a, b}$ \\ ${ }^{a}$ School of Pharmacy, Anhui Medical University; Hefei 230032, People's Republic of China: ${ }^{b}$ Key Laboratory of \\ Anti-inflammatory and Immune Medicines, Ministry of Education; Hefei 230032, People's Republic of China: and \\ ${ }^{c}$ Department of Pharmacy, Hospital of Armed Police of Anhui Province; Hefei 230032, People's Republic of China. \\ Received November 27, 2018; accepted May 20, 2019
}

Teriflunomide (TEF, A771726) is the active metabolite of leflunomide (LEF), a disease-modifying antirheumatic drug. The main purpose of this study was to develop and evaluate water-in-oil (W/O) microemulsion formulation of TEF. The W/O microemulsion was optimized formula is the physical and chemical

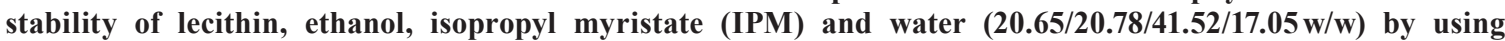
the pseudo-ternary phase diagram and the average droplet size is about $40 \mathrm{~nm}$. The permeability of TEF microemulsion is about 6 times higher than control group in vitro penetration test. The results of antiinflammatory effect showed that compared with the control group, the external TEF microemulsion group could significantly inhibit swelling of paw in rats, and no significant difference compared with oral LEF group. The results of hepatotoxicity test show that there were normal content of alanine aminotransferase (ALT)/aspartate aminotransferase (AST) and no obvious inflammatory infiltration of TEF microemulsion group compared with LEF group. The plasma concentration curve showed that compared with LEF group, the peak concentration of TEF microemulsion group was decreased, the half-life $\left(t_{1 / 2}\right)$ was prolonged, and the relative bioavailability of TEF microemulsion was $75.35 \%$. These results suggest that TEF W/O microemulsion can be used as a promising preparation to play an anti-inflammatory role while significantly reducing hepatotoxicity.

Key words teriflunomide; mircoemulsion; transdermal delivery; hepatotoxicity; pharmacokinetic

\section{Introduction}

Rheumatoid arthritis (RA) is an autoimmune disease. It is characterized by recurrent inflammation of the joints and surrounding tissues and leading to bone erosion and joint dysfunction. It is one of the world's refractory diseases. ${ }^{1,2)}$ Leflunomide (LEF), a new type of immunomodulator, has been commonly used alone or in combination with methotrexate for the treatment of RA as a kind of disease-modifying anti-rheumatic drugs (DMARDs). ${ }^{3)}$ LEF is a derivative of isoxazole, which is converted to teriflunomide (TEF, A771726) through the gastrointestinal tract and liver. It is an open loop structure with anti-inflammatory and immunosuppressive effects. ${ }^{3,4)}$ The metabolite of LEF to TEF occurs in the liver via two CYP enzymes, CYP1A2 and CYP2C19. The role model of TEF is considered to include the reversible enzyme dehydrogenase dehydrogenase (DHODH), which lowers the nucleotide level of pyrimidine and reduces the proliferation of activated T lymphocytes. ${ }^{4-6)}$ LEF in drug metabolism (>95\%) is almost entirely to the activity of compounds A771726 (Fig. 1), which cause the main pharmacodynamics of LEF, ${ }^{5,7)}$ Although LEF has been confirmed for the treatment of RA, serious liver injury and other adverse events have been reported frequently, and safety problems should also be paid attention. ${ }^{8)}$ In 2010, the food and drug administration (FDA) added a warning to the label of the drug, warning that there was a risk of severe liver damage on the label to monitor liver function during the treatment of LEF. The report says 49 cases of severe liver damage, including 14 severe liver failures, have been reported in the past seven years. Adverse events in the report highlight the risk of hepatotoxicity in patients with liver disease or elevated liver aminopherase. ${ }^{9)}$ We should pay more attention to its hepatotoxicity due to considering the wide use of LEF. Thus, it is necessary to consider the development of a new formulation to reduce the hepatotoxicity of LEF.

Transdermal drug delivery system (TDDS) is a kind of drugs through the skin to absorb dosing method, drug is applied to the skin, through the stratum corneum, to reach derma layer, again by capillary absorption into the systemic circulation process known as percutaneous absorption. It is one of the hotspots of drug preparation, which can avoid the first over effect of the liver and the degradation of the gastrointestinal tract, continuous administration, stabilize the plasma drug concentration, control the dosage and reduce the side effects of the drug. ${ }^{10,11)}$ Microemulsion, with droplet diameter ranges from 10 to $100 \mathrm{~nm}$, is by oil, water, surfactant, and helps the surfactant is mixed in certain proportion and the spontaneous formation of the isotropic, transparent, thermodynamic stability of the colloid dispersed system. ${ }^{12)}$ Nanoscale

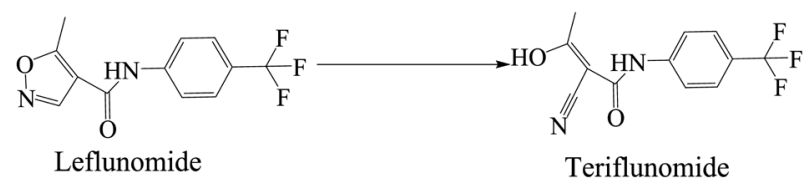

Fig. 1. Chemical Structure of Leflunomide and Its Active Metabolite Teriflunomide (A771726) 
microemulsion drops also make them suitable for dermal/topical administration, such as the water-in-oil (W/O) microemulsion has been used for transdermal absorption, including local transdermal absorption. ${ }^{13)}$ At the same time, microemulsion can promote the solubility of drugs, and the intercellular lipid bilayer of corneous layer has high compatibility, can penetrate the stratum corneum into the systemic circulation. The special structure of microemulsion droplet, the drug release time after percutaneous administration is longer, the blood drug concentration is more stable. ${ }^{14-17)}$ Therefore, microemulsion is often used as the carrier of TDDS.

Based on the above considerations, we think that it might be reasonable to reduce the hepatotoxicity of LEF by microemulsion. Thus, in our study, in order to reduce the hepatotoxicity of LEF, a stable W/O microemulsion was developed by using its active metabolite TEF, and its morphology, droplet size, $\mathrm{pH}$ value, conductivity, and stability were characterized. In addition, pharmacological studies were carried out to evaluate the anti-inflammatory and hepatotoxicity of microemulsion and the pharmacokinetics of TEF microemulsion to evaluate the potential of microemulsion as an effective transdermal delivery carrier of the drug.

\section{Experimental}

Materials TEF is homemade (purity is greater than 90\%). TEF standard products are purchased from Shanghai Macklin biochemical technology Co., Ltd. Soybean lecithin with a $\mathrm{P}$ phosphatidyl choline (PC) content of $95 \%$ was obtained from Shanghai A.V.T Medical Technology Co., Ltd. (Shanghai, People's Republic of China). LEF is purchased by Suzhou Changzheng Xinkai Pharmaceutical Co., Ltd. (Developed by Xinkai, U.S.A.). Methanol, ethanol (medicinal grade) and isopropyl myristate (IPM) were provided by Sinopharm Group Chemical Reagent Co., Ltd. (Shanghai, People's Republic of China). Carrageenan purchased by aladdin reagent (Shanghai) Co., Ltd. Carboxymethylcellulose sodium (CMC) purchased from Tianjin Guangfu Co., Ltd. alanine aminotransferase (ALT)/ aspartate aminotransferase (AST) kit purchased from Nanjing Institute of Biotechnology. All other chemicals and solvents were of analytical grade and used as received.

Animals Rats (Sprague Dawley, SD) weighing in the range of 250-300 g (provided by the Central Animal Laboratory of Anhui Medical University) were used for this study with the permission from the institutional ethical committee of the Anhui Medical University.

Construction of Pseudo-ternary Phase Diagrams Soybean lecithin is a safe and biocompatible non-ionic hydrophilic surfactants, and ethanol, as a cosurfactant, they were chosen to be a mixture surfactant in microemulsion. In order to determine the concentration range of the existing microemulsion, a water titration method was used to establish a pseudo ternary phase diagram at room temperature $\left(25^{\circ} \mathrm{C}\right)$. The surfactant (soybean lecithin) and the co-surfactant (ethanol) were mixed with different weight ratio $\left(K_{\mathrm{m}}\right)$ of $1: 1,1: 2,2: 1,2: 3$ and $3: 1(\mathrm{w} / \mathrm{w})$ to be mixed in the electromagnetic agitator for $30 \mathrm{~s}$, and the mixed surfactant was obtained. For each phase diagram, the oil phase (IPM) and the surfactant mixture (Sm) were mixed, where the ratios of oil to $\mathrm{Sm}$ in the mixtures were varied from $9: 1$ to $1: 9(\mathrm{w} / \mathrm{w})$. In the agitation, distilled water was dripped into the oil phase. Then, the appearance and transparency of the mixture were visually inspected. The amount of double distilled water from the clarification to turbidity or turbidity to clarification was recorded in the preparation of microemulsion, while recored the quality of surfactant, co-surfactant and oil phase. Finally, the microemulsion domain is determined by the mass fraction of each component at the critical point. ${ }^{18-20)}$

Preparation of Microemulsion The pseudo-ternary phase diagram of the largest area in microemulsion was selected to determine the concentration range of the components in the microemulsion, and the composition of the final formula was selected from the microemulsion area. The microemulsion containing TEF (A771726) was prepared. After the drug was dissolved in the distilled water, the drug solution was dripped into the oil phase containing the surfactant and the co-surfactant at room temperature. Then, a clear, transparent, homogeneity and fluidity of TEF microemulsion can be obtained.

\section{Characterization of TEF Microemulsion}

\section{Extrinsic Feature of Microemulsion}

The microemulsion was examined the color, homogeneity, isotropy, phase separation and fluidity by visual inspection at room temperature.

\section{Morphology of Microemulsion}

The microscopic morphology of microemulsion was detected by transmission electron microscope (TEM, Tecnai, TF20). Firstly, the microemulsion diluted by ethanol is dripped into the carbon coated copper net, then dried slightly, then $2 \%$ phosphotungstic acid (PTA) is used for negative staining at room temperature, and the appearance of the microemulsion can be measured after the sample was dried.

Droplet Size and Polydispersity Index (PDI)

The average PDI of microemulsion was measured by dynamic light scattering (DLS), using the Zetasizer Nano ZS90 (Malvern Instrument, Worcestershire, U.K.). The microemulsion samples were loaded into the cuvettes with all around light in the thermostated scattering chamber and measured at $25^{\circ} \mathrm{C}$ (set parameters such as viscosity $(6.02 \mathrm{mPa} \cdot \mathrm{s})$, temperature $\left(25^{\circ} \mathrm{C}\right)$ and refractive index (1.434)). The results of the measurement were triplicated.

$\mathrm{pH}$ Measurements

The $\mathrm{pH}$ values of the microemulsion samples were measured via $\mathrm{pH}$ meter (Leici Instrument Co., Shanghai, China) at room temperature and carried out triplicate measurements.

Conductivity Measurements

The electroconductibility of the samples was determined by DDS-11AW conductivity meter equipped with platinum electrode (Bante Instrument Co., Shanghai, China). Calibrate the conductivity cell used standard $\mathrm{KCl}$ solution prior to measurement of conductivity. Sample was measured for triplicate at ambient temperature.

Measurement Content of TEF (A771726)

The method of HPLC was used for the drug loading content determination. Samples directly injected to HPLC system without further treatment after diluted with mobile phase of methanol. A Shimpack ODS C18 column (Shimadzu Corporation, Kyoto, Japan), $5 \mu \mathrm{m}$ particle size, $250 \mathrm{~mm}$ _x0005_4.6mm, was used as stationary phase. Methanol and phosphate buffer were used as the mobile phase at a flow rate of $1.0 \mathrm{~mL} / \mathrm{min}$. During the detection process, the UV detection wavelength was set at $294 \mathrm{~nm}$ and the temperature of the chromatographic column was set at $40^{\circ} \mathrm{C}$. 


\section{Stability}

The $5 \mathrm{~mL}$ microemulsion samples were prepared and stored for three months at room temperature. During this period, the physical changes of the sample are checked regularly, including color changes, phase separation, clarification of microemulsion, and drug precipitation or content reduction. In addition, in order to evaluate the accelerated physical stability of microemulsion, the samples were centrifuged at $1000 \mathrm{rpm}$ for $30 \mathrm{~min}$, and the physical instability such as phase separation and aggregation was observed.

Ex Vivo Skin Permeation Studies SD rats weighing in the range of $250-300 \mathrm{~g}$ were used for the in vitro skin permeation study with the permission from the institutional ethical committee of the Anhui Medical University. After the rats were sacrificed, the abdominal hair was removed by electric shaving machine. Obtained the abdominal skin and the stripped off subcutaneous tissue by operation. The skin was washed with normal saline and sealed with tin foil. The abdominal skin could be stored at $-20^{\circ} \mathrm{C}$ and be used within a week. In vitro permeation experiments adopts Franz diffusion tank. The abdominal skin of rats was fixed between the supply pool and the receiving pool, and the keratinized layer was placed between the supply pool and the receiving pool and the dermis was attached to the receiving solution. The receiving solution was $0.01 \%$ phosphate buffer $(\mathrm{pH} 7.4) 17.8 \mathrm{~mL}$ and the effective osmotic area of the diffusion pool was $2.58 \mathrm{~cm}^{2}$. The receiver fluid was stirred with a magnetic rotor at a speed of $400 \mathrm{rpm}$ and maintained at $37^{\circ} \mathrm{C}$ in order to keep the skin surface at $32^{\circ} \mathrm{C}^{21)}$; An amount of $3 \mathrm{~mL}$ of $0.1 \%$ TEF solution, $0.1 \%$ TEF emulsion and $0.1 \%(\mathrm{w} / \mathrm{w})$ TEF microemulsion was placed into the donor compartment. Ensure that no air bubbles remained in the device. A $2 \mathrm{~mL}$ sample of the receptor fluid was collected at 2, 4, 6, 8, 12 and $24 \mathrm{~h}$ and immediately replaced by fresh solution. Samples were centrifuged under $15000 \mathrm{rpm}$ for $5 \mathrm{~min}$ and and all samples were filtered through a $0.45 \mu \mathrm{m}$ microporous filter membrane analysed for TEF by HPLC. All experiments were performed in triplicate.

The cumulative amount of TEF that permeated through the skin $\left(\mu \mathrm{g}^{*} \mathrm{~cm}^{-2}\right)$ was plotted as a function of time $(t)$ for each formulation. The rate of drug permeation $\left(J_{\mathrm{SS}}\right)$ was determined from the slope of the linear portion of the cumulative drug permeation versus time plot. Cumulative bioactive ingredient (TEF) permeation $\left(Q_{\mathrm{t}}, \mu \mathrm{g}\right)$ across the skin was calculated according to the following equation:

$$
Q_{\mathrm{t}}=V_{\mathrm{r}} C_{\mathrm{t}}+\sum_{i=0}^{t-1} V_{\mathrm{s}} C_{\mathrm{i}}
$$

where $C_{\mathrm{t}}\left(\mu \mathrm{g}^{*} \mathrm{~mL}^{-1}\right)$ is the TEF concentration of the receptor compartment medium at each sampling time, $C_{\mathrm{i}}\left(\mu \mathrm{g}^{*} \mathrm{~mL}^{-1}\right)$ is the TEF concentration of the $i$ th sample, and $V_{\mathrm{r}}(\mathrm{mL})$ and $V_{\mathrm{s}}(\mathrm{mL})$ are the volumes of the receptor medium and sample respectively. The permeability coefficient $\left(K_{\mathrm{p}}, \mathrm{cmh}^{-1}\right)$ was calculated by dividing the steady-state flux $\left(J_{\mathrm{SS}}\right)$ by the initial concentration of drug in the donor cell $\left(C_{0}\right)$, as shown by the following equation:

$$
K_{\mathrm{p}}=\frac{J_{\mathrm{ss}}}{C_{0}}
$$

where $C_{0}\left(\mathrm{mg}^{*} \mathrm{~mL}^{-1}\right)$ is the concentration of the bioactive ingredient in the donor compartment.

\section{Preclinical Pharmacology Studies}

Study of Anti-inflammatory and Hepatotoxicity Paw Edema Volume Test.

Establishment of inflammatory model by the used of carrageenan $(1 \%, 0.15 \mathrm{~mL})$ was injected via the intraplantar route. ${ }^{22)}$ The rats were divided into four groups: blank microemulsion group, CMC group, oral LEF group $(1 \% \mathrm{CMC}$ as suspending agent) and external TEF microemulsion group respectively. The right paw volume was measured before modeling, and successive medication $(2.1 \mathrm{mg} / \mathrm{kg})$ for six days and carrageenan $(1 \%)$ was injected via the intraplantar route to produce an inflammatory model in the sixth days of administration. A plethysmometer (Chengdu Tai Meng Software Co., Ltd,.) was used to measure the paw volume of the rats and paw edema volume each group was compared in. Results were expressed as:

$$
\text { Edema volume }=V_{\mathrm{t}}-V_{\mathrm{c}}
$$

$V_{\mathrm{t}}$ is paw volume $(\mathrm{mL})$ at time $\mathrm{t}$ after carrageenan administration. $V_{\mathrm{c}}$ is paw volume $(\mathrm{mL})$ before carrageenan administration.

Aspartate Aminotransferase (AST) and Alanine Aminotransferase (ALT) Index Detection

The rats were randomly divided into three groups, which were divided into control group, LEF group and TEF microemulsion group. Blood was taken from the eyelids after administration and plasma was retained after centrifugation was used to determine AST and ALT. The colorimetric kit was used according to the manufacturer's instructions.

Pathological Examination of Hepatotoxicity

The rats were randomly divided into three groups: LEF group, TEF microemulsion group and control group. The LEF group was given oral administration and the TEF microemulsion group was transdermal administration $(2.1 \mathrm{mg} / \mathrm{kg})$, the diet was normal. After $30 \mathrm{~d}$, the rats were sacrificed and their livers were taken out for pathological observation by hematoxylin-eosin (HE) staining.

Animal Pharmacokinetic Study

Animals were classed into four groups having 6 animals per group. The rats were given the drug according to their weight $(2.1 \mathrm{mg} / \mathrm{kg})$. Initial two groups respectively were given microemulsion of TEF and excluding TEF microemulsion. The third group was given the LEF by oral administration, and the last group was the blank control group. Blood samples were collected into heparinized tubes at $0,0.5,1,2,3,4,5,6,7,8$, $10,12,14,16,24,36$ and $48 \mathrm{~h}(n=6)$, and blood from a retroorbital vein. After taking the blood, centrifuge $(3800 \mathrm{r} / \mathrm{min}$, $10 \mathrm{~min}$ ) was taken to remove the supernatant, and the plasma was obtained $-20^{\circ} \mathrm{C}$ was stored until analysis by HPLC.

\section{HPLC Assay of Plasma Samples}

At the time of analysis, $200 \mu \mathrm{L}$ plasma was taken and $10 \mu \mathrm{L}$ internal standard $[\rho($ methyl- $p$-hydroxy benzoate $)=100 \mathrm{mg} / \mathrm{L}]$ was added and the vortex $30 \mathrm{~s}$. Then $1 \mathrm{~mL}$ of acetonitrile was added and the vortex for $3 \mathrm{~min}$. After completely extraction, it was centrifuged for $5 \mathrm{~min}(15000 \mathrm{r} / \mathrm{min})$, and the supernatant of the mixture was blown to dried at $40^{\circ} \mathrm{C}$. The residue was dissolved in the mobile phase of $200 \mu \mathrm{L}$. After centrifugation $(5 \mathrm{~min} ; 15000 \mathrm{r} / \mathrm{min})$ the supernatant of $20 \mu \mathrm{L}$ was sucked with a pipette and analyzed by HPLC. A Shimpack ODS C18 column (Shimadzu Corporation, Kyoto, Japan), $5 \mu \mathrm{m}$ particle size, 250 mm_x0005_4.6mm was used as stationary phase. 

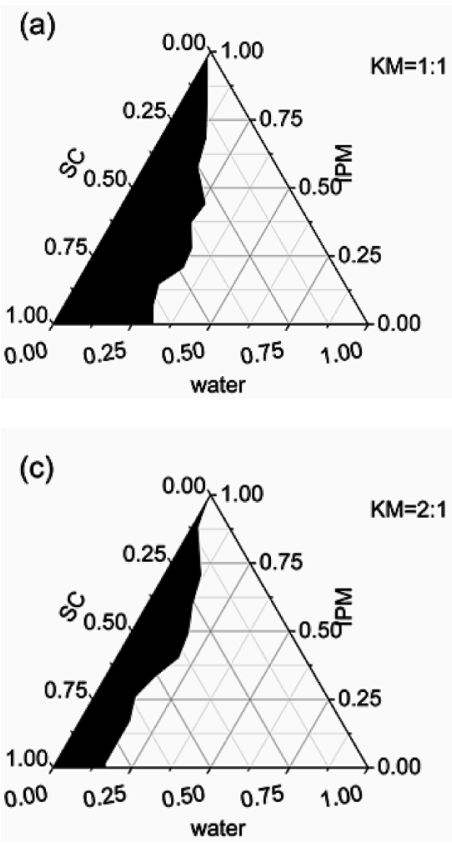

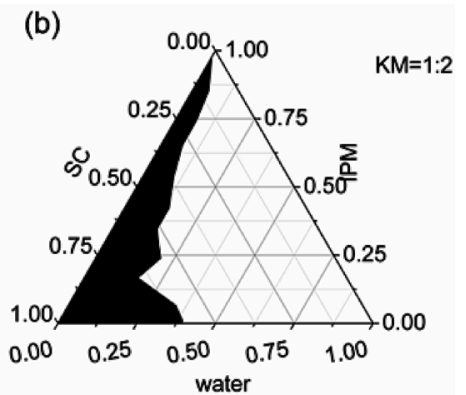

(d)

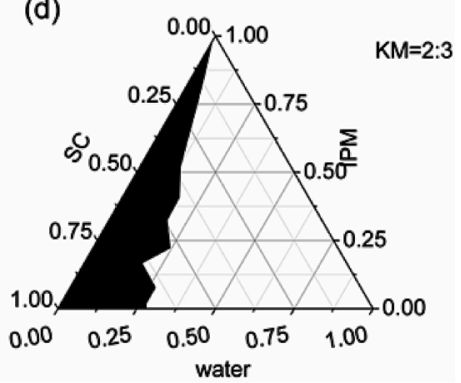

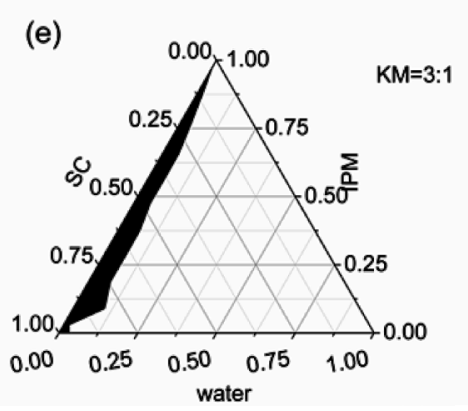

Fig. 2. Pseudo-ternary Phase Diagrams Composed of Lecithin, Ethanol, Isopropyl Myristate and Water at Different $K_{\mathrm{m}}$ (a) $1: 1$, (b) $1: 2$, (c) $2: 1$, (d) $2: 3$ and (e) $3: 1$

Methanol and $1 \%$ phosphate buffer $(\mathrm{pH}$ 7.4) were used as the mobile phase at a flow rate of $1.0 \mathrm{~mL} / \mathrm{min}$. The relevant parameter settings include the detection wavelength of $294 \mathrm{~nm}$ and the column temperature of $40^{\circ} \mathrm{C}$. In the concentration range of $0.0625-3.125 \mathrm{mg} / \mathrm{L}$ and the concentration of TEF was determined by standard curve.

Pharmacokinetic Analysis

The main pharmacokinetic parameters in the two groups of oral LEF and external TEF microemulsion were calculated by DAS 3.0 (Drug and Statistics, Shanghai University of Traditional Chinese Medicine, Shanghai, China). The analysis data included plasma concentration $\left(C_{\max }\right)$, peak time $\left(T_{\max }\right)$, area under the curve $(A U C)$, half-life $\left(t_{1 / 2}\right)$ and relative bioavailability. The relative bioavailability of the TDDS was calculated as follows:

$$
\text { Relative bioavailability }=\frac{(A U C) \text { microemulsion }}{(A U C) \text { leflunomide }} \times 100 \%
$$

Statistical Analysis All data were analyzed by one-way ANOVA. The differences were considered statistically significant when $p<0.05$.

\section{Results and Discussion}

Pseudo-ternary Phase Diagram Study and Preparation of Microemulsion Microemulsion is a transparent, thermo- dynamic stability and isotropic oil and water mixing system, which is generally maintained stability by surfactant and cosurfactant. Soybean lecithin as a natural ionic surfactant (In general, soybean lecithin is classified as amphoteric surfactant too) with excellent performance has many functional characteristics such as emulsification, softening, wetting, dispersing, penetration, innocuity, no irritation and no pollution. It has phosphatidylcholine (PC) content of $(>95 \%)$. It can enhance the bioavailability of the object molecules by enhancing the permeability of permeable drugs and transmembrane channels across the gastrointestinal tract. ${ }^{23-25)}$ The reason why ethanol was chosen as the preparation of microemulsion is that it reduces the polarity of the aqueous phase, increases the mobility and elasticity of the interfacial film, and improves the defect that the phospholipid forming boundary film is rigid and easy to form gel or liquid crystal state, which is conducive to the formation of microemulsion. ${ }^{26,27)}$ In addition, it has been found that a mixture of lecithin and ethanol can form a W/O microemulsion with high water content. ${ }^{28)}$ Therefore, in this study, we chose lecithin, ethanol, IPM and water W/O microemulsion formulations, which are all acceptable in pharmacy and were used to improve the percutaneous absorption of drugs. ${ }^{29)}$

Five pseudo-ternary phase diagrams were constructed as described in Experimental by titration with water. Figure 2 depicts the pseudo-ternary phase diagram of lecithin and 
ethanol with different weight ratios $\left(K_{\mathrm{m}}\right)$. The shadow section in the figure represents the region of the existence of stable, clear and transparent W/O microemulsion. As shown in Fig. 2, the area of the microemulsion decreases with the increase of $K_{\mathrm{m}}$ from $1: 1$ (a) to $3: 1$ (e), and the area of the microemulsion obtained under the ratio of lecithin/ethanol to $1: 1(\mathrm{w} / \mathrm{w})$ is the largest. When $K_{\mathrm{m}}$ was fixed at $1: 2$ (b), 2:1 (c), and 2:3 (d), the area of the three microemulsion areas had little difference and all were less than $K_{\mathrm{m}}(1: 1)$. The region of microemulsion decreases sharply when $K_{\mathrm{m}}$ was fixed at $3: 1$ (d). Lecithin is insoluble in water and only in the water swelling, gel concentration, so the microemulsion region is decreased probably due to the concentration of lecithin mixed surfactant increased in liquid crystal region increases, and the amount of surfactant may cause a series of problems, such as security and the increase in the viscosity of microemulsion decreased. ${ }^{30,31)}$ On the basis of considering these factors the $K_{\mathrm{m}} 1: 1$ was selected to prepare drug loading $\mathrm{W} / \mathrm{O}$ microemulsion.

As shown in Fig. 2 (a), a clear and transparent microemulsion was found in the microemulsion area. Therefore, according to the principle of high drug loading efficiency and low proportion of surfactant on mucosal toxicity the final formula was $20.17 \%$ lecithin, $20.31 \%$ ethanol, $41.52 \%$ IPM and $17.05 \%$ water selected from the region.

Characterization of Microemulsion The microemulsion formulas shown in Fig. 3 are homogeneous, transparent, no precipitation, good fluidity, homogeneous, and yellow at room temperature. The microemulsion morphology observed by transmission electron microscopy (TEM) is shown in Fig. 4. Transmission electron microscope photographs show that the microemulsion droplets have a spherical appearance and uniform size. It can enhance the stability of microemulsion, improve flocculation, coalescence and precipitation when the drop diameter of microemulsion is small. ${ }^{32}$ As shown in Fig. 5 , the average droplet size of microemulsion about $40 \mathrm{~nm}$ with Zetasizer APS. The different physico-chemical parameters of the microemulsion were characterized and the results were shown in Table 1. The data in the table showed that compared with the particle size of blank microemulsion, drug-containing microemulsion particle size was not significantly with the drug content of $0.05,0.1,0.25 \%(\mathrm{w} / \mathrm{w})$. Yet compared with blank microemulsion the partical was significantly larger when the content increased to $0.5 \%(p<0.01)$ maybe the drug loading was to large of microemulsions. In this study, the PDI value with a polydispersity index of less than 0.5 proved that a homogeneous microemulsion was obtained. ${ }^{33)}$ In addition, the $\mathrm{pH}$ values observed are considered physiologically acceptable and incorporation of the drug caused no significant change. In the case of conductance, the results show that the microemulsion was W/O type because of the oil has a low conductivity
(In terms of conductivity, the results show that microemulsion is oil in water because its conductivity is very low and its conductivity is close to $0 \mathrm{~S} / \mathrm{cm}) .{ }^{34,35)}$ In microemulsion, the concentration of TEF remained almost unchanged and the size of the small liquid droplets changed little, no degradation and precipitation or color change was not observed. There were

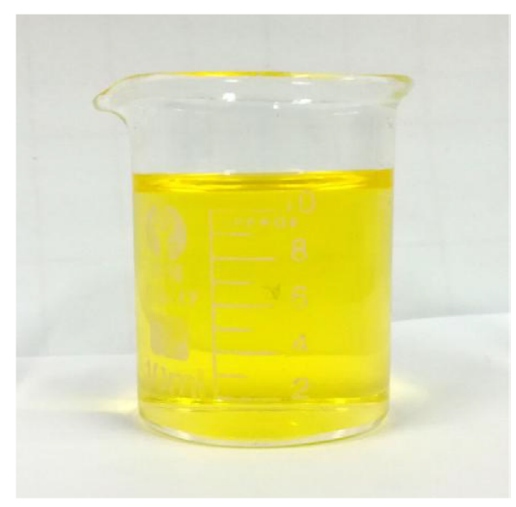

Fig. 3. The Appearance of Microemulsion (Color figure can be accessed in the online version.)

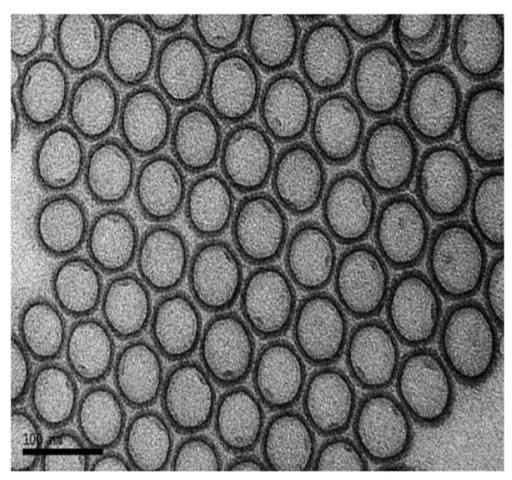

Fig. 4. Transmission Electron Microphotography (TEM) of Teriflunomide Microemulsion

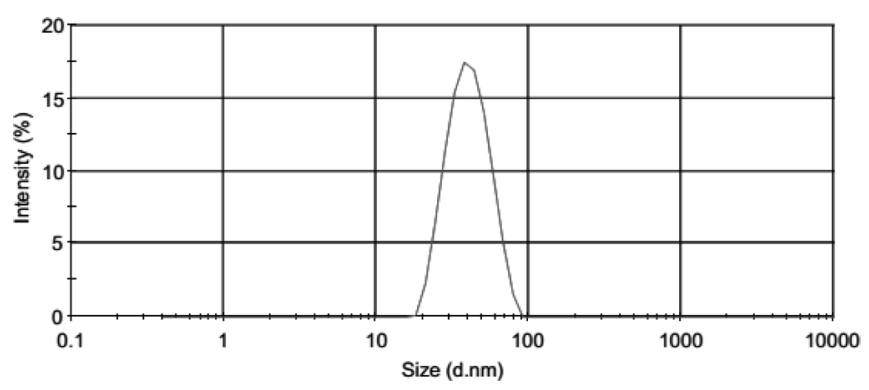

Fig. 5. The Size Distribution of Teriflunomide Microemulsion

Table 1. Characterization Parameters of Optimized Microemulsion

\begin{tabular}{lccccc}
\hline \hline & & \multicolumn{3}{c}{ TEF ME } \\
\cline { 3 - 6 } Parameter formulation & Blank ME & $0.05 \%$ & $0.10 \%$ & $0.25 \%$ & $0.50 \%$ \\
\hline pH & $6.130 \pm 0.078$ & $6.310 \pm 0.020$ & $6.240 \pm 0.020$ & $6.330 \pm 0.044$ & $6.360 \pm 0.075$ \\
PDI & $0.187 \pm 0.017$ & $0.206 \pm 0.030$ & $0.272 \pm 0.056$ & $0.198 \pm 0.031$ & $0.259 \pm 0.025$ \\
Droplet size $(\mathrm{nm})$ & $35.680 \pm 1.040$ & $41.290 \pm 10.681$ & $43.210 \pm 1.928$ & $42.860 \pm 3.516$ & $108.640 \pm 14.122 * *$ \\
EC $(\mu \mathrm{s} / \mathrm{cm})$ & $3.364 \pm 0.259$ & $1.049 \pm 0.113$ & $2.843 \pm 0.005$ & $4.690 \pm 0.010$ & $5.180 \pm 0.138$ \\
\hline
\end{tabular}

ME: microemulsion; PDI: Polydispersity; EC: Electrical conductivity. **Significantly different from that of comper with blank ME groups $(p<0.01)$. 
no obvious change in the determination of drug content (no data), indicating that the microemulsion was stable under the above conditions. In addition, no signs of phase separation or breakage or drug precipitation were observed after centrifugation, indicating that the preparation was physically stable for centrifugation.

Ex Vivo Skin Permeation Studies As shown in Fig. 6, the permeability difference between TEF microemulsion, TEF solution and TEF emulsion with a content of $0.1 \%(\mathrm{w} / \mathrm{w})$ was compared. There percentage cumulative was a significant difference between TEF solution and microemulsion $(p<0.01)$ and the difference between TEF microemulsion and TEF emulsion was obvious $(p<0.01)$ also. The enhancement of permeability of TEF microemulsion may be related to the affinity of the ester in the surfactant and the diameter of the nanoparticles. Better permeability of TEF microemulsion may be caused by its nano-droplet size that is nanodroplet size presented a large surface-to-volume ratio, enabling large amounts of bioactive ingredient molecules to get access to the skin in the surface-to-surface interaction between the skin and the microemulsion. ${ }^{36)}$ Little TEF was detected in the receptor medium, demonstrating that TEF in emulsion form could difficult to permeate through the skin layer.

Furthermore, the $K_{\mathrm{p}}$ value of TEF microemulsion $(0.1 \%$ $(\mathrm{w} / \mathrm{w}))$ was found to be $\left(6.1592 \times 10^{-4}\right) \mathrm{cm} \mathrm{h}^{-1}$ significantly than that TEF solution $\left(0.1 \% \quad(\mathrm{w} / \mathrm{w}) \quad\left(0.5608 \times 10^{-4}\right) \mathrm{cm} \mathrm{h}^{-1}\right.$ $(p<0.05)$. However, the $K_{\mathrm{p}}$ value of the emulsion was $0.9690 \times 10^{-4} \mathrm{~cm} \mathrm{~h}^{-1}$, which was significantly different from that of TEF microemulsion. As we all know, the main component of cell membrane is soybean phospholipid, which can fuse with cuticle increase its fluidity and promote skin penetration. In addition, the droplet size and viscosity of the dosage form can affect its efficiency. The small droplet size and low viscosity of microemulsion make it an excellent carrier for enhancing percutaneous absorption of TEF, because the number of vesicles that can interact with the cuticle fixed region. When the droplet size and viscosity decrease, the stratum corneum will increase. ${ }^{37)}$ Therefore, the interaction between surfactants and hydrophilic lipophilic structures formed by cosurfactant and the minimum droplet size of microemulsions may be the possible reasons for the penetration enhancement of TEF from microemulsions.

\section{Preclinical Pharmacology Studies}

Study of Anti-inflammatory

The paw edema volume was shown in Fig. 7. Compared with the CMC group, the foot swelling of the microemulsion group was significantly reduced, the results were significantly different $(p<0.05)$, and there was no significant difference between the LEF group and the microemulsion group. One percent CMC can be used as LEF suspension aid without affecting its efficacy or interfering with the experimental results. $^{22)}$ The results showed that the external of TEF microemulsion could reduce the swelling of the rat paw, which the microemulsion could effectively infiltrate the skin into the blood circulation.

Hepatotoxicity Study

The results of Table 2 showed the activity of AST and ALT in the control group and the experimental group. The results showed that there was no significant difference between the TEF microemulsion group and the control group. Compared with the LEF group the difference was significant. The

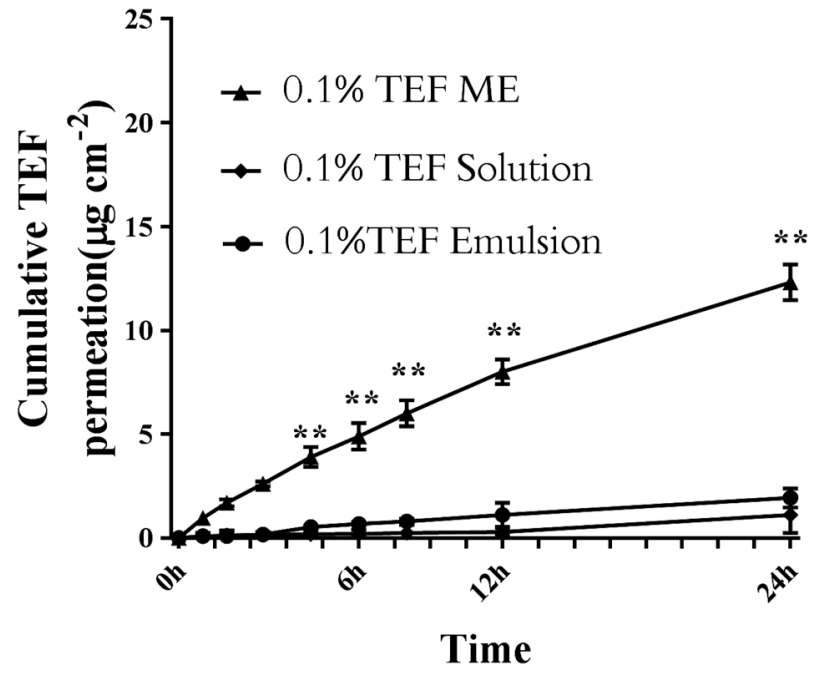

Fig. 6. Ex Vivo Skin Permeation Studies of 0.1\%TEF Microemulsion, $0.1 \%$ TEF Solution, $0.1 \%$ TEF Emulsion $(n=3)$

The data was analysed using one-way ANOVA. **Significantly different from that of compered with TEF solution groups $(p<0.01)$.

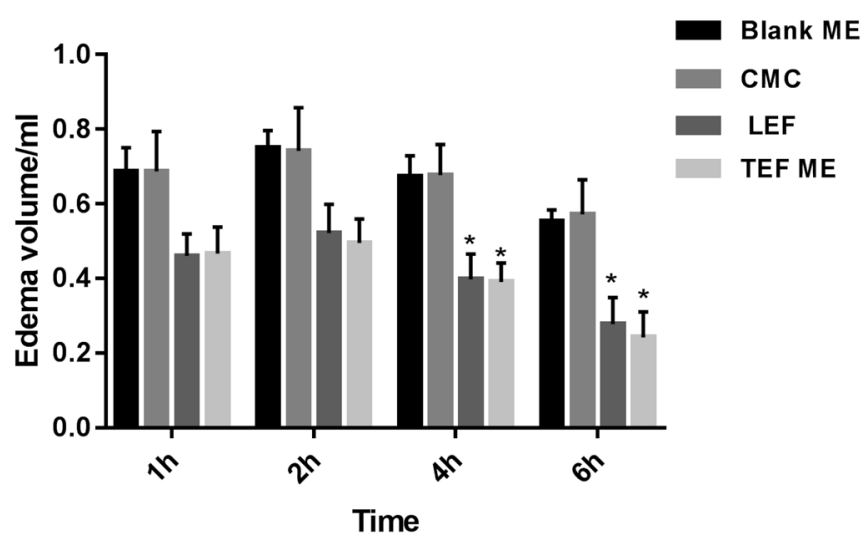

Fig. 7. Experiment of Paw Swelling in Rats: Results Are Presented as Mean \pm S.E.M. $(n=6)$

The data was analysed using one-way ANOVA followed by Dunnett test. $* p<0.05$ when compared with control group (CMC and blank ME groups). Blank ME: given blank microemulsion through the skin; CMC: oral carboxymethylcellulose sodium; LEF: oral leflunomide solution; TEF ME: given TEF microemulsion through the skin.

Table 2. Serum AST, ALT Activities in Experimental Animals (Mean \pm S.D., $n=6$ )

\begin{tabular}{cccc}
\hline \hline Parametes & Control & Leflunomide & TEF ME \\
\hline AST & $19.13 \pm 2.15$ & $45.02 \pm 9.97^{*}$ & $27.69 \pm 2.75$ \\
ALT & $22.04 \pm 4.36$ & $49.88 \pm 7.47^{*}$ & $26.01 \pm 3.93$ \\
\hline
\end{tabular}

*Significantly different from that of comper with control groups $(p<0.05)$.

AST/ALT content in LEF group was significantly increased, while TEF microemulsion group were not. The results showed that external of TEF microemulsion in vitro had little damage to the liver.

The pathological section of rat liver was shown in Fig. 8. In the TEF group, the inflammatory infiltration in the LEF group was obvious compared with the LEF group. A large number of inflammatory cells with darker nuclei and less cytoplasm visible in LEF group and there was no visible inflammation 


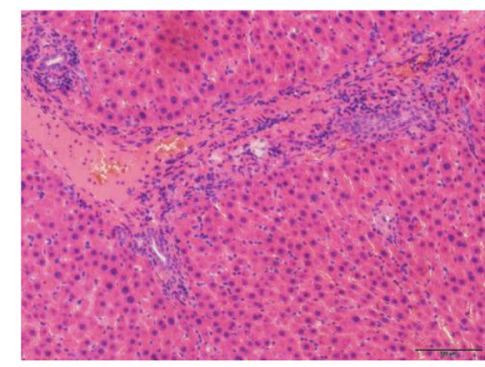

(a)

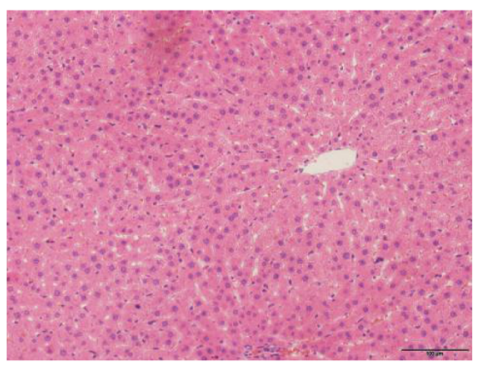

(b)

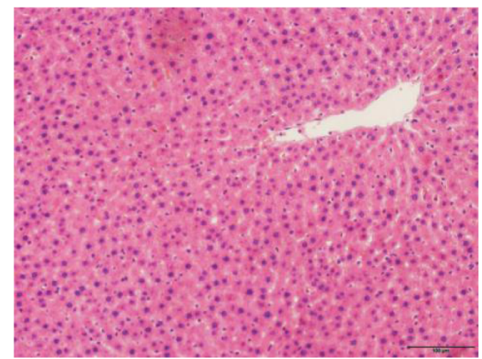

(c)

Fig. 8. The Results of Pathological Section in Hepatic Tissue

(a) was leflunomide oral administration group; (b) was TEF microemulsion group, no obvious pathological changes in liver cells; (c) was control group, and liver cells were in normal state. (Color figure can be accessed in the online version.)

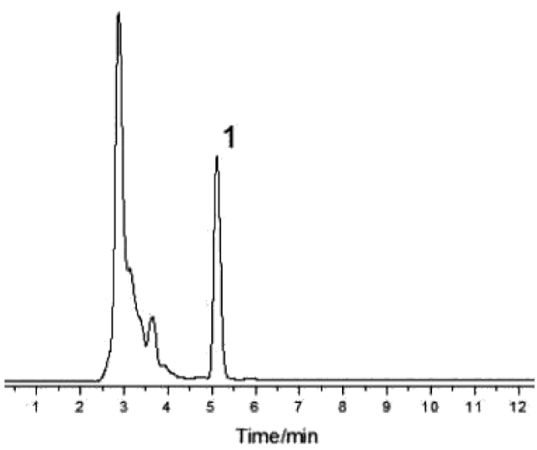

(a)

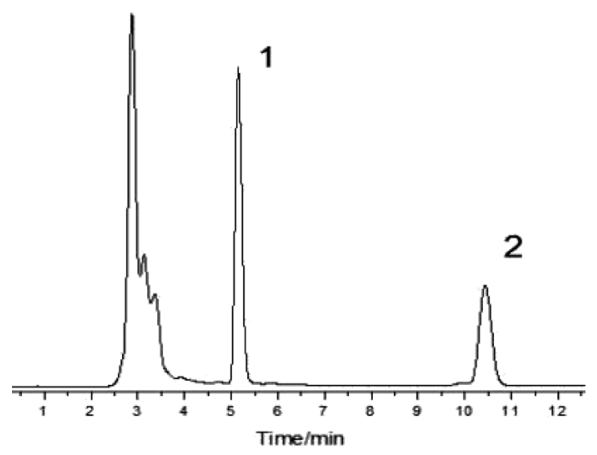

(c)

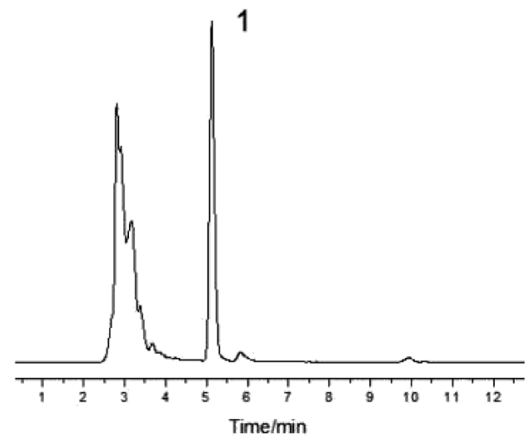

(b)

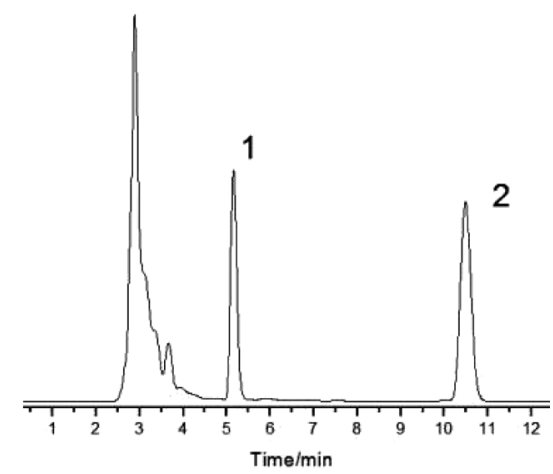

(d)

Fig. 9. Representative HPLC Chromatograms of (a): Blank Plasma; (b): Blank Microemulsion; (c): TEF Microemulsion and (d): Oral Leflunomide 1: Methylparaben (Internal Standard Substance); 2: TEF 
in the TEF microemulsion group. There was no significant change in liver slices compared with normal group. The results showed that chronic oral LEF had a certain degree of hepatotoxicity, while TEF microemulsion group and normal control group had no obvious damage. From the above results, it is speculated that TEF microemulsion can achieve the desired therapeutic effect and reduce the hepatotoxicity of LEF by topical administration.

Pharmacokinetic Studies in Rats TEF representative chromatograms in rat plasma (containing methyl paraben) as shown in Fig. 9. Impurity peaks were observed in blank plasma (a) at $3 \mathrm{~min}$, methyl paraben (internal standard) at $5 \mathrm{~min}$, and in blank microemulsion group (b) at $5 \mathrm{~min}$. Both TEF microemulsion group (c) and oral LEF group (d) showed a standard peak of TEF at $10 \mathrm{~min}$, which was no significant interference was observed in the retention time of TEF between the internal standard. Figure 10 shows that the plasma concentration-time profiles of group of LEF suspension and TEF microemulsion at a dosage of $2.1 \mathrm{mg} / \mathrm{kg}$ body weight. The plasma concentration of TEF in the microemulsion group was lower than that in the oral group due to the change of the drug delivery pathway. The pharmacokinetic parameters of TEF are listed in Table 3. The result shows that $A U C_{0 \rightarrow \infty}$ values of oral LEF was $27.09 \pm 8.42 \mathrm{~h}^{*} \mathrm{mg} / \mathrm{L}$ and that of the TEF microemulsion was $20.42 \pm 7.81 \mathrm{~h} * \mathrm{mg} / \mathrm{L}$, yielding a relative bioavailability of $75.35 \%$. TEF microemulsion was absorbed and reached a $C_{\max }$ of $1.33 \pm 0.13 \mathrm{mg} / \mathrm{L}$ at a $T_{\max }$ of $7.5 \pm 1.76 \mathrm{~h}$. Whereas, the $C_{\max }$ for the LEF suspension was $2.77 \pm 0.51 \mathrm{mg} / \mathrm{L}$ at a $T_{\max }$ of $4.50 \pm 0.55 \mathrm{~h}$. Obvious double peak phenomenon was observed in two groups indicating that has hepatic enteric circulation. In addition, the results showed that the $t_{1 / 2}$ of TEF microemulsion was longer than LEF, the $T_{\max }$ time was delayed and the $C_{\max }$ was smaller, but after $15 \mathrm{~h}$ of plasma TEF concentration was higher than oral LEF group suggesting that microemul-

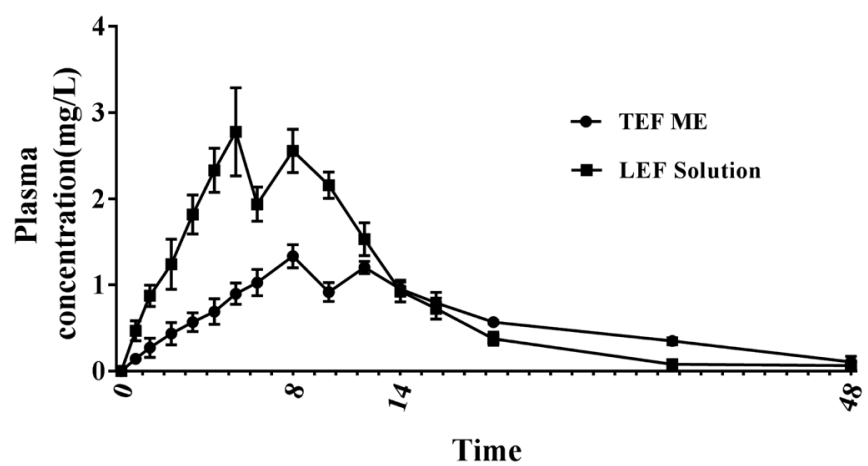

Fig. 10. Mean Plasma Concentration-Time Profiles of TEF in Rats after LEF Solution by Oral Administration and Given TEF Microemulsion through the Skin

Table 3. Pharmacokinetic Parameters after TEF ME and Oral Administration of Leflunomide (Mean \pm S.D., $n=6$ )

\begin{tabular}{lcc}
\hline \hline Parametes & Leflunomide & \multicolumn{1}{c}{ TEF ME } \\
\hline$T_{\max }(\mathrm{h})$ & $4.50 \pm 0.55$ & $7.5 \pm 1.76^{*}$ \\
$C_{\max }(\mathrm{mg} / \mathrm{L})$ & $2.77 \pm 0.51$ & $1.33 \pm 0.13^{*}$ \\
$T_{1 / 2}(\mathrm{~h})$ & $9.38 \pm 2.45$ & $13.10 \pm 2.02^{*}$ \\
$A U C_{0 \rightarrow \infty}(\mathrm{h} * \mathrm{mg} / \mathrm{L})$ & $27.09 \pm 8.42$ & $20.42 \pm 7.81$ \\
Relative bioavailability $(\%)$ & - & 75.35 \\
\hline
\end{tabular}

*Significantly different from that of compered with leflunomide groups $(p<0.05)$. sion might have the effect of drug storage and so release of TEF was slow.

As a promising TDDS, microemulsion is used as a continuous phase to prepare $\mathrm{W} / \mathrm{O}$ microemulsion, which is compatible with the sebum of hair follicle. The particle size of microemulsion transdermal delivery carrier is similar to the size of hair follicle, which can promote the penetration of hydrophilic drugs through the hair follicle into the skin. In addition, lipids and surfactants in microemulsions can increase membrane fluidity, thereby improving the permeability of hydrophilic drug molecules, promoting cross cell absorption and opening tight junctions between cells to allow cell side transport. ${ }^{38,39)}$ In our study, the bioavailability of TEF microemulsion was lower than that of LEF, but percutaneous administration can effectively make the drug enter the systemic circulation. Therefore, due to the accumulation effect of the skin, the fluctuation of the blood concentration of the drug is small, and it can steady stable blood concentration for a long time. It is speculated that the hepatotoxicity of the drug may be reduced, perhaps because of its effect avoided the liver first pass effect and the accumulation of liver metabolism through the skin thereby reduced the hepatotoxicity. In addition, this is conducive to topical treatment of arthritis that the topical use of TEF microemulsion can play a continuous role and can also be discontinued at any time. These results suggest that TEF W/O microemulsion can be used as a promising preparation to play an anti-inflammatory role while significantly reducing hepatotoxicity. Moreover, TEF microemulsion is only a preliminary exploration to avoid hepatotoxicity, and the related research may not be comprehensive enough, including the local application of its transdermal preparation needs further study.

\section{Conclusion}

In order to reduce the hepatotoxicity of LEF, the microemulsion of oil in water was used as a transdermal drug delivery system. A stable W/O TEF microemulsion formulation consists of soybean lecithin, anhydrous ethanol, IPM and water $(20.65 / 20.78 / 41.52 / 17.05 \mathrm{w} / \mathrm{w})$ was successfully obtained and the average droplet size was about $40 \mathrm{~nm}$. Based on the findings of our study, it was concluded that microemulsion containing TEF, could be used as potential drug delivery system for topical and TDDS.

Acknowledgments This work was supported by Anhui Province Natural Science Foundation (No.1808085MH235) and Scientific Research Foundation of Institute of Anhui Province Transformational Medicine (No. 2017zhyx32).

Conflict of Interest The authors declare no conflict of interest.

Supplementary Materials The online version of this article contains supplementary materials. As the reviewer mentioned, this study supplements the cryo-TEM images (Due to the limited conditions of this unit, it was specially photographed at University of Science and Technology of China). The image is not very clear because of too long exposure time can cause blistering. This is mainly due to the fact that the sample is easy to melt or foam when the electron hits the prepared sample. What we can see is that the sample is dark and the irregular round droplets can be seen. 


\section{References}

1) Shimomura S., Inoue H., Arai Y., Nakagawa S., Fujii Y., Kishida T., Ichimaru S., Tsuchida S., Shirai T., Ikoma K., Mazda O., Kubo T., Int. J. Mol. Sci., 19, 1653 (2018).

2) Aletaha D., Neogi T., Silman A. J., et al., Ann. Rheum. Dis., 62, 2569-2581 (2010).

3) Kis E., Nagy T., Jani M., Molnár E., Jánossy J., Ujhellyi O., Német K., Herédi-Szabó K., Krajcsi P., Ann. Rheum. Dis., 68, 1201-1207 (2009).

4) Vrenken T. E., Buist-Homan M., Kalsbeek A. J., Faber K. N., Moshage H., J. Hepatol., 49, 799-809 (2008).

5) Breedveld F. C., Dayer J.-M., Ann. Rheum. Dis., 59, 841-849 (2000).

6) Jiang L., Zhang W., Li W., Ling C., Jiang M., Toxicol. Lett., 10, 1-37 (2017).

7) Ma Z.-G., Zhang X., Yuan Y.-P., Jin Y.-G., Li N., Kong C.-Y., Song P., Tang Q.-Z., Clin. Sci., 132, 685-699 (2018).

8) Wang L., Ma L., Lin Y., Liu X., Xiao L., Zhang Y., Xu Y., Zhou H., Pan G., Mol. Pharmacol., 93, 563-574 (2018).

9) U.S FDA, "FDA Drug Safety Communication: New boxed warning for severe liver injury with arthritis drug Arava (LEF).": 〈https: //www.fda.gov/Drugs/DrugSafety/Postmarket Drug Safety Information for Patientsand Providers/ucm218679. Htm> 2010.

10) Soni M., Kumar S., Gupta G. D., J. Pharm. Res., 2, 1184-1190 (2009).

11) Shishu, Kamalpreat, Maheshwari M., Drug Development and Pharmaceutical Industry, 38, 294-300 (2012).

12) Shannon P., Int. J. Pharm., 425-442 (2017)

13) Okur N. Ü., Yavaşoğlu A., Karasulu H. Y., Chem. Pharm. Bull., 62, 135-143 (2014).

14) Negi P., Singh B., Sharma G., Beg S., Raza K., Katare O. P., Drug Deliv., 23, 951-967 (2016).

15) Kogan A., Garti N., Adv. Colloid Interface Sci., 123-126, 369-385 (2006).

16) Sintov A. C., Int. J. Pharm., 481, 97-103 (2015).

17) Zhang J., Michniak-Kohn B., Int. J. Pharm., 421, 34-44 (2011).

18) Chen H., Chang X., Weng T., Zhao X., Gao Z., Yang Y., Xu H., Yang X., Control Release, 98, 427-436 (2004).

19) Kumar B., Jain S. K., Prajapati S. K., International Journal of Drug Delivery, 3, 83-94 (2011).
20) Shishu, Rajan S., Kamalpreet, AAPS PharmSciTech, 10, 559-565 (2009).

21) Hong L., Zhou C.-L., Chen F.-P., Han D., Wang C.-Y., Li J. X., Chi Z., Liu C.-G., J. Microencapsul., 10, 1-13 (2017).

22) Brito A. M. S., Godin A. M., Augusto P. S. A., Menezes R. R. Melo I. S. F., Dutra M. G. M. B., Costa S. O. A. M., Goulart F. A., Rodrigues F. F., Isis Morais M., Machado R. R., Coelho M. M., Eur. J. Pharmacol., 818, 17-25 (2018).

23) Kirjavainen M., Urtti A., Mönkkönen J., Hirvonen J., Eur. J. Pharm. Sci., 10, 97-102 (2000).

24) Wu H., Lu C., Zhou A., Min Z., Zhang Y., Drug Deve. Ind. Pharm., 35, 138-144 (2009).

25) Lin C. C., Lin H. Y., Chi M. H., Shen C. M., Chen H. W., Yang W. J., Lee M. H., Food Chem., 154, 282-290 (2014).

26) Shinoda K., Araki M., Sadaghiani A., Khan A., Lindman B., J. Chem. Phys., 95, 989-993 (1991).

27) Lee P. J., Langer R., Shastri V. P., Pharm. Res., 20, 264-269 (2003).

28) Avramiotis S., Bekiari V. V., Lianos P., Xenakis A., J. Colloid Interface Sci., 194, 326-331 (1997).

29) Lin H., Michniak-Kohn B., Xia Z., Xu L., Kang Q., Chen C., Ma S., Wu Q., Curr. Drug Deliv., 15, 1-11 (2018).

30) Von Corswant C., Engstrom S., Soderman O., Langmuir, 13, 50615070 (1997)

31) Man Xu Q. Y. M., Yu, Q., Zhao Q., Chen W., Lin Y., Jin Y., Drug Development and Pharmaceutical Industry, 42, 280-287 (2016).

32) Cho Y. H., Kim S., Bae E. K., Mok C. K., Park J., J. Food Sci., 73 , E115-E121 (2008).

33) Biruss B., Dietl R., Valenta C., Chem. Phys. Lipids, 148, 84-90 (2007).

34) Peira E., Scolari P., Gasco M. R., Int. J. Pharm., 226, 47-51 (2001).

35) Kong M., Chen X. G., Kweon D. K., Park H. J., Carbohydr. Polym., 86, 837-843 (2011).

36) Ki-Taek Kim K. T., Kim M. H., Park J. H., Lee J. Y., Cho H. J., Yoon I. S., Kim D. D., J. Ginseng Res., 42, 512-523 (2018).

37) Mishra R., Prabhavalkar K. S., Bhatt L. K., J. Liposome Res., 26, 297-306 (2016)

38) Constantinides P. P., Pharmacol. Res., 12, 1561-1572 (1995).

39) Sha X., Yan G., Wu Y., Li J., Fang X., Eur. J. Pharm. Sci., 24, 477-486 (2005). 